\title{
Feasibility of Extracted-Overlay Fusion Imaging for Intraoperative Treatment Evaluation of Radiofrequency Ablation for Hepatocellular Carcinoma
}

\author{
Yuki Makino ${ }^{a}$ Yasuharu Imai ${ }^{a}$ Takumi Igura ${ }^{a}$ Sachiyo Kogita ${ }^{a}$ \\ Yoshiyuki Sawai $^{a}$ Kazuto Fukuda ${ }^{a}$ Takayuki Iwamoto ${ }^{a}$ \\ Junya Okabe ${ }^{a}$ Manabu Takamura ${ }^{b}$ Norihiko Fujita $^{b}$ \\ Masatoshi Horic Tetsuo Takeharad ${ }^{d}$ Masatoshi Kudoe \\ Takamichi Murakami ${ }^{f}$
}

Departments of a Gastroenterology and ${ }^{b}$ Radiology, Ikeda Municipal Hospital, Ikeda, Departments of 'Radiology and dGastroenterology and Hepatology, Osaka University Graduate School of Medicine, Suita, Departments of ${ }^{e}$ Gastroenterology and Hepatology and ${ }^{f}$ Radiology, Kinki University Faculty of Medicine, Osaka-Sayama, Japan

\section{Key Words}

Contrast-enhanced ultrasonography · Extracted-overlay fusion imaging ·

Hepatocellular carcinoma · Multimodality fusion imaging $\cdot$ Radiofrequency ablation

\begin{abstract}
Background and Aims: Extracted-overlay fusion imaging is a novel computed tomography/ magnetic resonance-ultrasonography (CT/MR-US) imaging technique in which a target tumor with a virtual ablative margin is extracted from CT/MR volume data and synchronously overlaid on US images. We investigated the applicability of the technique to intraoperative evaluation of radiofrequency ablation (RFA) for hepatocellular carcinoma (HCC). Methods: This retrospective study analyzed 85 HCCs treated with RFA using extracted-overlay fusion imaging for guidance and evaluation. To perform RFA, an electrode was inserted targeting the tumor and a virtual 5-mm ablative margin overlaid on the US image. Following ablation, contrast-enhanced US (CEUS) was performed to assess the ablative margin, and the minimal ablative margins were categorized into three groups: (I) margin $<0 \mathrm{~mm}$ (protrusion), (II) margin 0 to $<5 \mathrm{~mm}$, and (III) margin $\geq 5 \mathrm{~mm}$. Margin assessment was based on the positional relationship between the overlaid tumor plus margin and the perfusion defect of the ablation
\end{abstract}


zone. Tumors in group I underwent repeat ablation until they were in groups II or III. The final classifications were compared with those obtained by retrospectively created fusion images of pre- and post-RFA CT or MR imaging (CT-CT/MR-MR fusion imaging). Results: Treatment evaluation was impossible using CEUS in six HCCs because the tumors were located far below the body surface. Of the remaining $79 \mathrm{HCCs}$, the categorizations of minimal ablative margins between CEUS extracted-overlay fusion imaging and CT-CT/MR-MR fusion imaging were in agreement for 72 tumors (91.1\%) (Cohen's quadratic-weighted kappa coefficient 0.66, good agreement, $p<0.01)$. Conclusions: Extracted-overlay fusion imaging combined with CEUS is feasible for the evaluation of RFA and enables intraoperative treatment evaluation without the need to perform contrast-enhanced CT.

Copyright (C) 2016 S. Karger AG, Basel

\section{Introduction}

The introduction of multimodality fusion imaging technology has improved the effectiveness of radiofrequency ablation (RFA) for hepatocellular carcinoma (HCC). Computed tomography/magnetic resonance-ultrasonography (CT/MR-US) fusion imaging, which enables the synchronous display of real-time US images and cross-sectional multiplanar reconstruction CT or MR images, is reportedly useful for RFA treatment guidance [1-7]. Moreover, although pre- and post-RFA CT or MR images have been conventionally compared side-byside to evaluate therapeutic responses, pre- and post-RFA CT-CT/MR-MR fusion images have made it possible to more accurately measure minimal ablative margins [8-14].

The CT/MR-US extracted-overlay fusion imaging technique was developed recently [15]. In this novel technique, a target tumor with a virtual ablative margin is extracted from CT/MR volume data and synchronously overlaid on US images (fig. 1). By virtue of the clear visualization of the tumor and ablative margin, this technique provides effective RFA treatment guidance. This technique has potential for evaluation of RFA treatment by combination with contrast-enhanced US (CEUS) [16-18]. In the present study, we analyzed the feasibility of extracted-overlay fusion imaging for intraoperative and quantitative RFA treatment evaluation, and compared the results with those of CT-CT/MR-MR fusion imaging.

\section{Patients and Methods}

\section{Patients and Tumors}

Our institutional review board approved this retrospective study, and informed consent was waived. The two inclusion criteria were as follows: (1) HCCs that underwent radical RFA using a Cool-tip RF Ablation System (Covidien, Boulder, CO, USA) and extracted-overlay fusion imaging from March 2013 to January 2014, and (2) HCCs for which CT-CT/MR-MR fusion imaging was available for RFA treatment evaluation using CT or MR images taken $\leq 2$ months before RFA and within 1 month after RFA. Of 101 consecutive patients with 139 HCC nodules who underwent RFA during that period, 68 patients with 85 HCCs that met the inclusion criteria were analyzed. The patients' clinical characteristics are presented in table 1. The diagnosis of HCC was established based on typical contrast-enhanced CT or MR imaging features [19]. When typical imaging findings were not observed, a tumor biopsy was performed to confirm the diagnosis of HCC.

CT/MR-US Fusion Imaging and Extracted-Overlay Fusion Imaging

Volume Navigation (GE Healthcare, Milwaukee, WI, USA) installed in a LOGIQ E9 imaging system (GE Healthcare) was used for CT/MR-US fusion imaging. The imaging modality that most clearly depicted the target tumor was used to produce the reference images. For extracted-overlay fusion imaging, a tumor was segmented from CT or MR imaging volume data and a 5-mm virtual ablative margin was added 

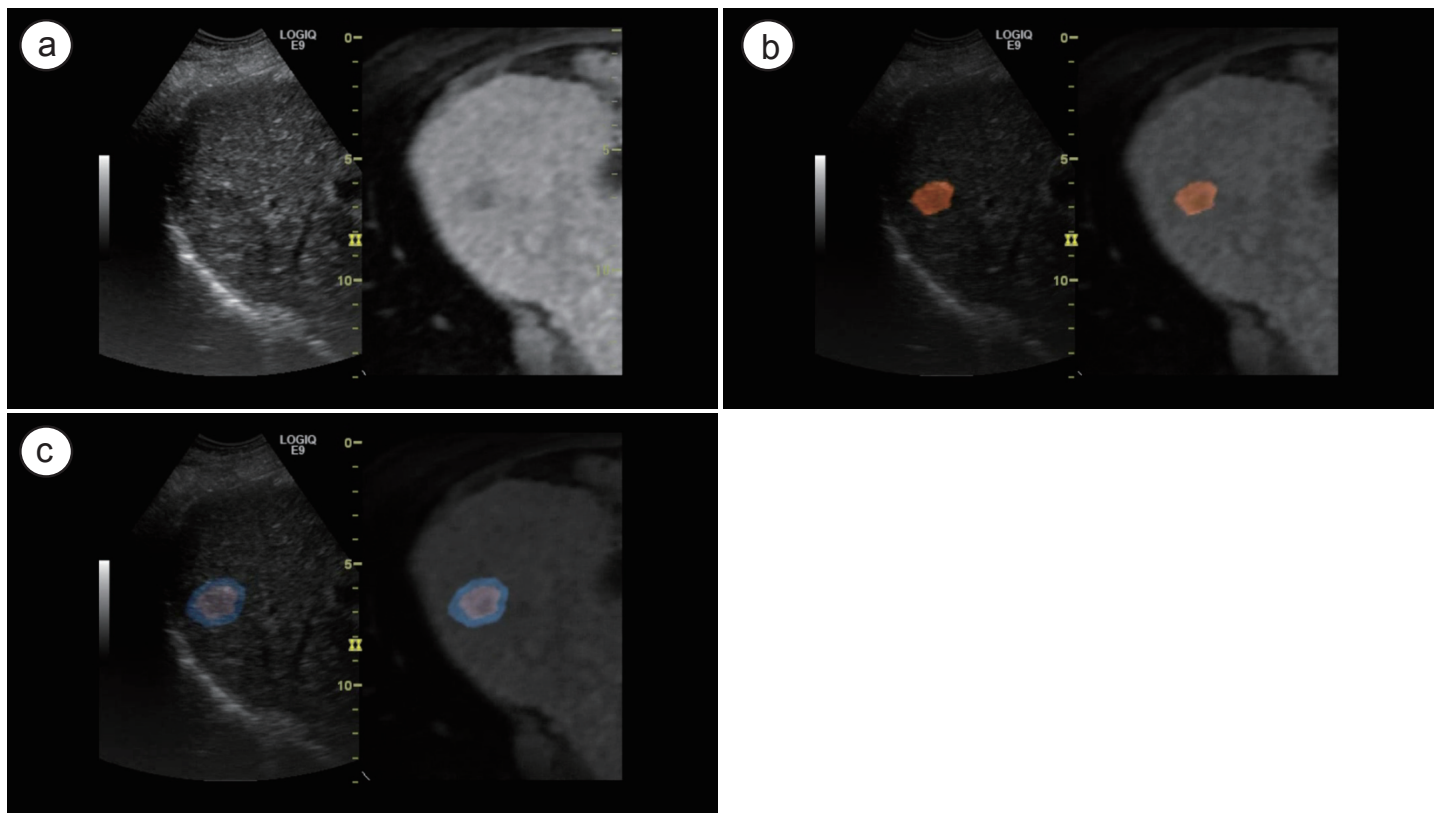

Fig. 1. Extracted-overlay fusion imaging in a 66-year-old woman with HCC in segment VIII. The tumor identified on the hepatobiliary-phase Gd-EOB-DTPA-MR image was used as the reference image and was overlaid on the real-time ultrasonography (US) image. a Conventional MR-US fusion imaging. b The extracted-overlay fusion image overlies the target tumor only. $\mathbf{c}$ The extracted-overlay fusion image overlies the target tumor (pink portion) and a 5-mm virtual ablative margin (blue portion) is added.

Table 1. Characteristics of 68 patients with 85 HCCs treated with RFA using extracted-overlay fusion imaging

\begin{tabular}{ll}
\hline Characteristic & Value \\
\hline Age (years) & $76(52-89)^{\dagger}$ \\
Gender (male/female) & $41 / 27$ \\
Etiology (HBV/HCV/others) & $9 / 52 / 7$ \\
Tumor diameter (mm) & $11.9(4.4-37.0)^{\dagger}$ \\
Tumor vascularity (hypervascular/hypovascular) & $75 / 10$ \\
Imaging modalities used for the extracted-overlay function & $10 / 2 / 7 / 5 / 1 / 60$ \\
(arterial phase of dynamic CT/portal phase of dynamic CT/CTHA/CTAP/ & \\
arterial phase of Gd-EOB-DTPA-enhanced MR imaging /hepatobiliary & \\
phase of Gd-EOB-DTPA-enhanced MR imaging) & $74 / 11$ \\
Size of the exposed tip of the Cool-tip needle (2 cm/3 cm) & $2(1-5)^{\dagger}$ \\
Number of ablations per tumor at the first treatment session & $80 / 4 / 1$ \\
Number of treatment sessions per tumor before complete ablation (1/2/3) & $17(3-27)^{\dagger}$ \\
Follow-up period (months) & \\
\hline
\end{tabular}

†Median (range). HBV=hepatitis B virus; HCV=hepatitis C virus; CTHA=CT during hepatic arteriography; CTAP=CT during arterial portography.

to the segmented tumor on an image processing workstation (Advantage Workstation VolumeShare 4; GE Healthcare). These procedures have been described in detail in the literature $[3,5,15]$. 


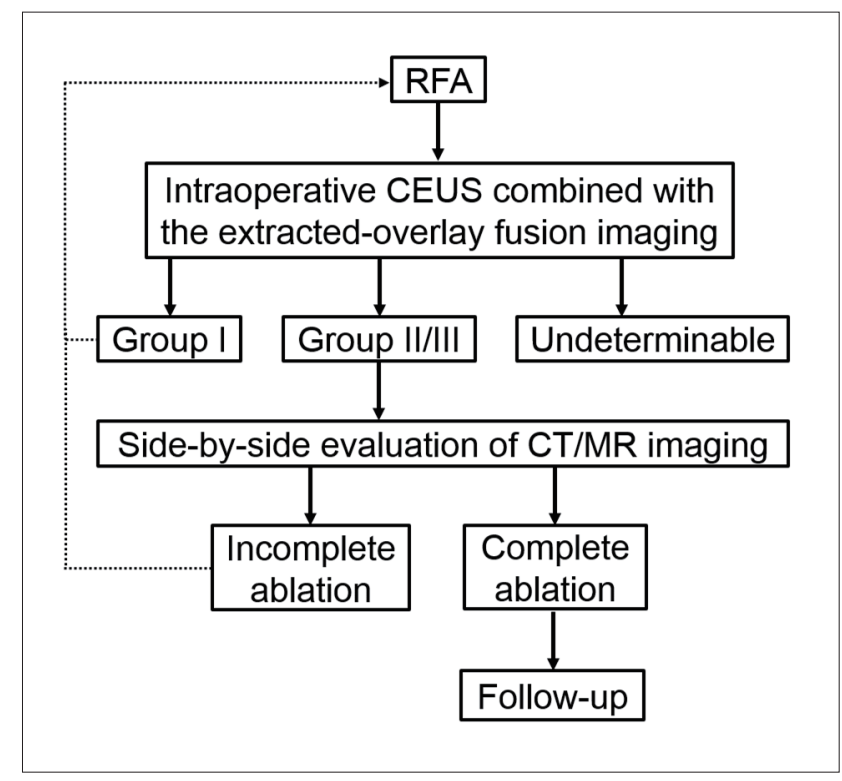

Fig. 2. Flow chart of the treatment procedures in this study.

RFA Procedures and Intraoperative Treatment Evaluation by CEUS Using Extracted-Overlay Fusion Imaging

A flow chart of the treatment and evaluation procedures is presented in fig. 2. One of three hepatologists with more than 10 years' experience performed the RFA procedure using a single 2- or 3-cm Cool-tip electrode. Using extracted-overlay fusion imaging, an electrode was inserted, the overlaid image of the tumor with a 5-mm margin was targeted, and ablation was conducted. Overlapping ablation was added as necessary. Following ablation, the therapeutic effect was assessed by CEUS using $0.0075 \mathrm{ml} / \mathrm{kg}$ of perfluorobutane microbubble (Sonazoid; Daiichi-Sankyo, Tokyo, Japan). The perfusion defect of the ablation zone was scanned in the vascular phase (0-120 s after administration of Sonazoid), with the image of the tumor plus a 5-mm margin overlaid on the CEUS image. Any additional tumors were subsequently evaluated. The RFA operator classified the minimal ablative margins into three groups based on the positional relationship between the overlaid tumor plus margin and the perfusion defect: (I) margin $<0 \mathrm{~mm}$ (tumor extends outside the ablation zone), (II) margin 0 to $<5 \mathrm{~mm}$, and (III) margin $\geq 5 \mathrm{~mm}$ (fig. 3 ). The CEUS criterion for complete ablation was classification as group II or III. Tumors in group I underwent immediate repeat ablation until they were classified into group II or III.

\section{RFA Treatment Evaluation by Side-by-Side CT or MR Imaging and Subsequent Follow-Up}

The effectiveness of RFA treatment was evaluated by dynamic CT or gadolinium ethoxybenzyl diethylenetriamine pentaacetic acid-enhanced MR (Gd-EOB-DTPA-MR) imaging within 30 days after RFA. A radiologist with more than 10 years' experience in abdominal radiology compared the pre- and postRFA images side by side. The two criteria for complete ablation were no signs of early enhancement and circumferential extension of the ablation zone beyond the estimated tumor boundary. The tumor underwent repeat ablation until both criteria were satisfied. After complete ablation, a follow-up contrastenhanced CT/MR image was obtained every 3 to 4 months. Local tumor progression was defined as the appearance of early enhancement and washout adjacent to the ablation zone.

\section{Retrospective Treatment Evaluation by CT-CT/MR-MR Fusion Imaging}

One radiologist with more than 10 years' experience in abdominal radiology and who was blinded to the intraoperative CEUS data created CT-CT or MR-MR fusion images for this study. Minimal ablative margins were measured three-dimensionally and classified into three groups in the same manner as that done for intraoperative CEUS (fig. 4). The detailed procedures of fusion image creation have been described in previous reports $[8,13]$. 


\section{Liver \\ Cancer}
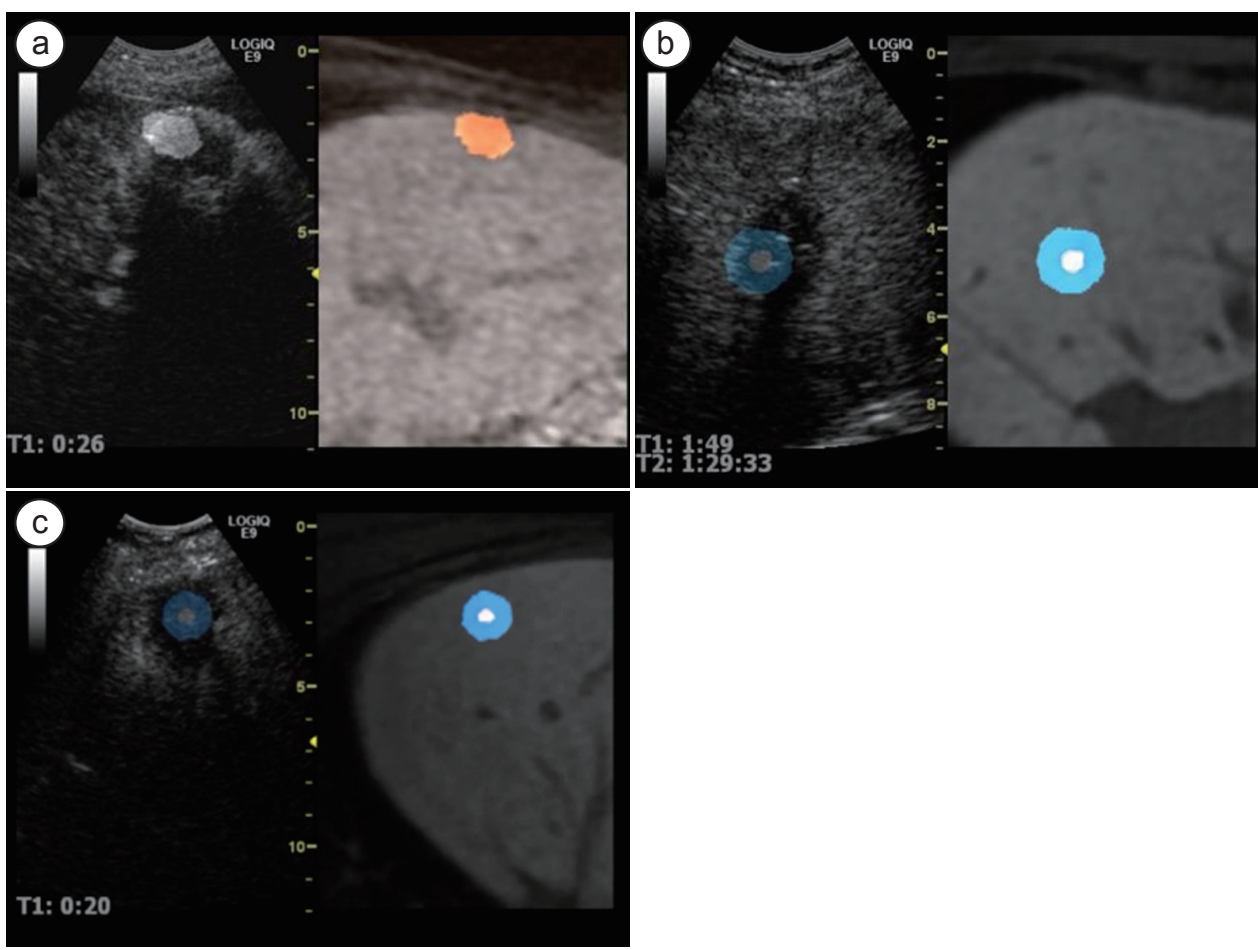

Fig. 3. Categorization of minimal ablative margins after RFA using extracted-overlay fusion imaging combined with CEUS. a Group I: margin $<0 \mathrm{~mm}$ (tumor extends outside the ablation zone); (b) group II: margin 0 to $<5 \mathrm{~mm}$; (c) group III: margin $\geq 5 \mathrm{~mm}$.

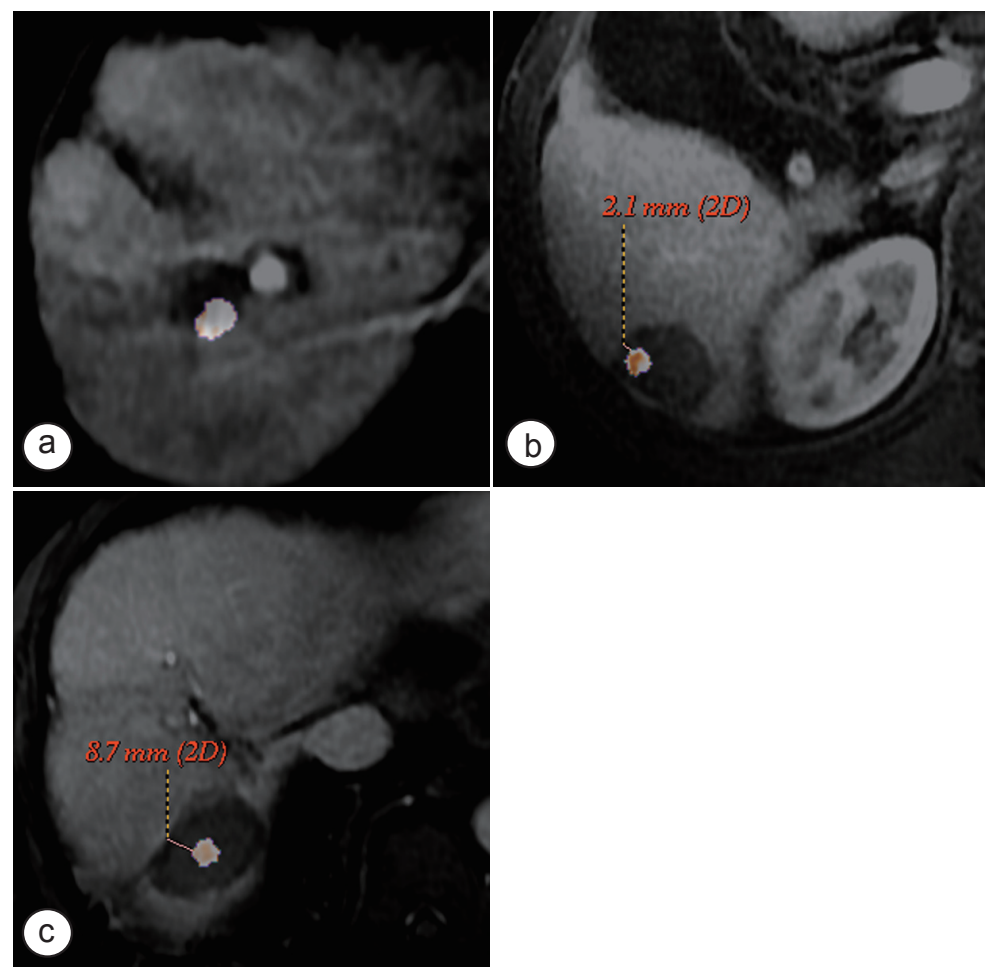

Fig. 4. Categorization of minimal ablative margins after RFA by CT-CT/MR-MR fusion imaging. a Group I: margin $<0 \mathrm{~mm}$ (tumor extends outside the ablation zone); (b) group II: margin 0 to $<5 \mathrm{~mm}$; (c) group III: margin $\geq 5 \mathrm{~mm}$. MR-MR fusion images are presented in this figure.

\section{KARGER}


Image Acquisition

Dynamic CT and CT angiography were performed using a 64-channel multidetector row helical CT system (Discovery CT 750HD; GE Healthcare) with slice thicknesses of 1.25 and $0.625 \mathrm{~mm}$, respectively. MR images were acquired with a 1.5-T MR system (Signa Excite HD 1.5T; GE Healthcare). The slice thicknesses of the dynamic- and hepatobiliary-phase Gd-EOB-DTPA-MR images were 3 or $5 \mathrm{~mm}$. The protocols for image acquisition have been described in the literature $[13,15]$.

\section{Statistical Analysis}

We evaluated the rate of concordance of the judgments of complete/incomplete ablation between intraoperative CEUS and side-by-side CT/MR evaluation after RFA. The overall agreement of the classification of the minimal ablative margin (i.e., into groups I, II, or III) between intraoperative CEUS and CT-CT/MR-MR fusion imaging was expressed by Cohen's quadratic-weighted kappa coefficient (poor agreement, $\mathrm{k}=0-0.20$; slight agreement, $\mathrm{k}=0.21-0.40$; moderate agreement, $\mathrm{k}=0.41-0.60$; good agreement, $\mathrm{k}=0.61-0.80$; excellent agreement, $\mathrm{k}=0.81-1.00$ ). The cumulative local tumor progression rate for each ablative margin group was estimated by the Kaplan-Meier method, and statistical significance was analyzed by the log-rank test. SPSS version 18.0 software (SPSS, Chicago, IL, USA) was used for all statistical analyses, and a p value of less than 0.05 was considered to be statistically significant.

\section{Results}

All patients were treated without severe complications. Moving images of the treatment procedures and intraoperative CEUS are presented in Electronic Supplementary Material 1 (for all online suppl.material, see www.karger.com/doi/10.1159/000443561). The preparation of extracted-overlay fusion imaging, including image processing on a workstation and image alignment on an US unit, took less than 25 min in all cases. Treatment evaluation using intraoperative CEUS took less than 10 min for all tumors.

\section{Treatment Evaluation of the First RFA Session}

In 6 of the 85 HCCs (7.1\%) the therapeutic response was impossible to evaluate because of CEUS signal attenuation problems with tumors located distant from the body surface. In the remaining 79 HCCs, the intraoperative CEUS evaluations were compared with both the side-by-side CT/MR evaluations and the retrospectively created CT-CT/MR-MR fusion imaging evaluations.

During intraoperative CEUS of the first RFA treatment session, the number of tumors evaluated as belonging to groups I, II, and III were 2 (2.5\%), 71 (89.9\%), and 6 (7.6\%), respectively. Complete ablation (groups II and III) was achieved in 77 HCCs (97.5\%) according to the CEUS criteria. It was impossible to perform additional ablation during the first treatment session for two HCCs in group I because of the patients' physical exhaustion. The median tumor diameters in groups I, II, and III were 16.1 (15.0-17.2) mm, 12.0 (4.4-37.0) mm, and $10.2(8.7-13.4) \mathrm{mm}$, respectively.

During the side-by-side CT/MR evaluation after the first treatment session, 75 of 79 HCCs (94.9\%) were judged to be completely ablated, and treatment was thus ended for these 75 patients. Four HCCs (5.1\%) were considered to be incompletely ablated. The judgment regarding complete or incomplete ablation was in agreement between CEUS and side-by-side CT/MR evaluation in 77 of 79 HCCs (97.5\%) (table 2).

Next, we retrospectively created CT-CT/MR-MR fusion imaging using images recorded before and after the first treatment session and categorized tumor ablation margins into three groups in the same manner as was done for CEUS. This new categorization was compared with that obtained by intraoperative CEUS after the first treatment session in 79 HCCs. The median registration error of CT-CT/MR-MR fusion imaging was $1.3 \mathrm{~mm}$ (range, 0.44$2.9 \mathrm{~mm}$ ), and the median minimal ablative margin was $1.6 \mathrm{~mm}$ (range, -28.3 to $10.4 \mathrm{~mm}$ ). 
Table 2. Judgment of complete or incomplete ablation by intraoperative CEUS with extracted-overlay fusion imaging and by side-by-side CT/MR evaluation

\begin{tabular}{lllll}
\hline & & \multicolumn{2}{l}{ Side-by-side CT/MR evaluation } & \\
& & Incomplete & Complete & Total \\
\hline CEUS & Incomplete & 2 & 0 & 2 \\
& Complete & 2 & 75 & 77 \\
& Total & 4 & 75 & 79 \\
\hline
\end{tabular}

Table 3. Categorization of minimal ablative margin by intraoperative CEUS with extracted-overlay fusion imaging and by CT-CT/MR-MR fusion imaging after the first treatment session

\begin{tabular}{llllll}
\hline & & \multicolumn{2}{l}{ CT-CT/MR-MR fusion imaging } & \\
& & Group I & Group II & Group III & Total \\
\hline CEUS & Group I & 2 & 0 & 0 & 2 \\
& Group II & 6 & 65 & 0 & 71 \\
& Group III & 0 & 1 & 5 & 6 \\
& Total & 8 & 66 & 5 & 79 \\
\hline
\end{tabular}

Group I: margin $<0$ mm (tumor extends outside the ablation zone); group II: margin 0 to $<5 \mathrm{~mm}$; group III: margin $\geq 5 \mathrm{~mm}$.

The numbers and median diameters of HCCs in groups I, II, and III were 8 (10.1\%) and 14.7 (6.7-19.7) mm, $66(83.5 \%)$ and $11.8(4.4-37.0) \mathrm{mm}$, and $5(6.3 \%)$ and $10.1(8.7-23.6) \mathrm{mm}$, respectively. The categorization of minimal ablative margins after the first treatment session assessed using intraoperative CEUS corresponded with that of CT-CT/MR-MR fusion imaging in 72 HCCs $(91.1 \%)$, and the overall agreement was good $(\mathrm{k}=0.66 ; 95 \%$ confidence interval, 0.42-0.90; $\mathrm{p}<0.01$ ) (table 3).

\section{Treatment Evaluation after the Final RFA Session and Cumulative Local Tumor Progression Rate}

After the first RFA treatment session, repeat ablation was conducted in four HCCs judged to be incompletely ablated by intraoperative CEUS and/or by side-by-side CT/MR evaluation (table 2), resulting in complete ablation based on both CEUS and side-by-side CT/MR criteria. After the final RFA treatment session, the numbers of tumors in groups I, II, and III were $0(0.0 \%), 73(92.4 \%)$, and $6(7.6 \%)$, respectively, based on intraoperative CEUS evaluation. The 1-year cumulative local tumor progression rates in groups II and III were $8.9 \%$ and $0.0 \%$, respectively (fig. 5).

\section{Discussion}

The effectiveness of RFA treatment has conventionally been assessed by side-by-side comparison of pre- and post-RFA CT images [8-14, 20]. However, because this side-by-side interpretation tends to be inaccurate, several new methods have been developed for more accurate treatment evaluation, such as nonenhanced MR imaging [21], superparamagnetic iron oxide-MR imaging [22, 23], Gd-EOB-DTPA-MR imaging [24], and iodized oil retention [25]. CT-CT/MR-MR fusion imaging was recently developed and is considered to be a useful imaging modality for RFA evaluation in terms of its accuracy, quantitative nature, noninvasiveness, and applicability to hypovascular lesions [8-14]. In the present study, we analyzed the clinical 


\section{Liver \\ Cancer}

Liver Cancer 2016;5:269-279

DOI: $10.1159 / 000449338$

Published online: September 14, 2016

(c) 2016 S. Karger AG, Basel www.karger.com/lic

Makino et al.: Extracted-Overlay Fusion Imaging for Evaluation of RFA

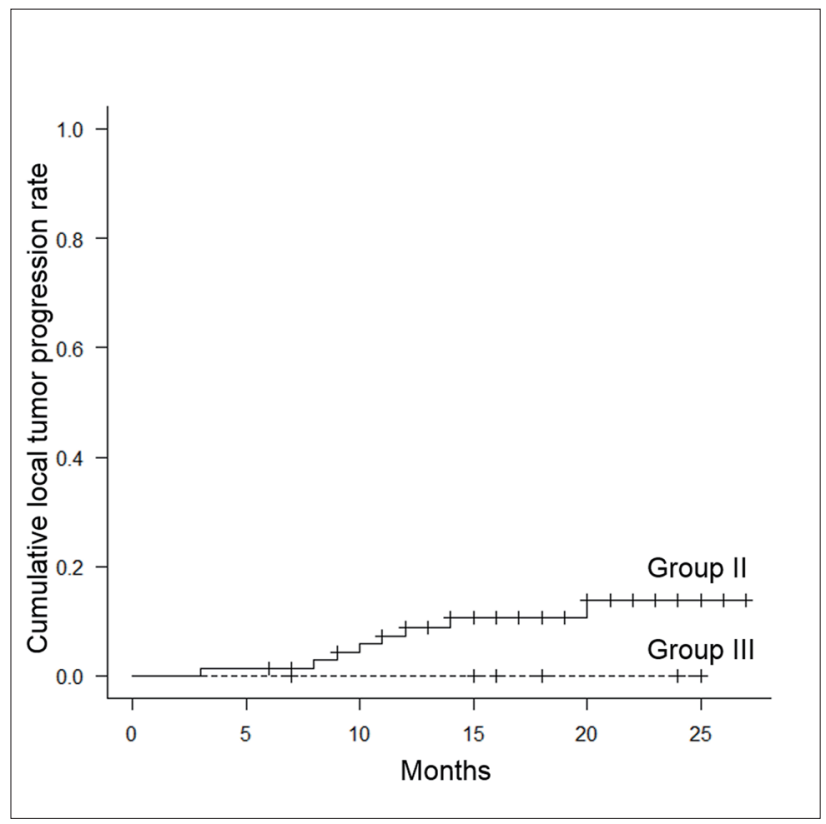

Fig. 5. Cumulative local tumor progression rate of two groups categorized by intraoperative CEUS using extracted-overlay fusion imaging. The 1-year cumulative local tumor progression rates in groups II and III were $8.9 \%$ and $0.0 \%$, respectively.

feasibility of extracted-overlay fusion imaging combined with CEUS, a novel CT/MR-US fusion imaging technique [15], for intraoperative RFA treatment evaluation.

By comparing intraoperative CEUS extracted-overlay fusion imaging evaluations with conventional side-by-side CT/MR evaluations, we investigated the concordance rate of the judgment of complete ablation. A high rate of concordance was observed between intraoperative CEUS and side-by-side CT/MR evaluations after RFA (table 2). Only 2 of 79 HCCs $(2.5 \%)$ judged to be completely ablated on CEUS were categorized as incompletely ablated according to the side-by-side CT/MR evaluation criteria and therefore required additional treatment sessions. These results suggest that intraoperative CEUS using extracted-overlay fusion imaging is reliable when used to determine the necessity of additional ablation.

We next analyzed the accuracy of the quantitative evaluation of the minimal ablative margin by comparison of intraoperative CEUS extracted-overlay fusion imaging with CT-CT/ MR-MR fusion imaging. The minimal ablative margins were categorized into three groups that are reportedly related to local tumor progression [8, 13, 20, 25]. Good agreement was observed between the evaluations based on intraoperative CEUS and CT-CT/MR-MR fusion imaging (table 3). The cumulative local tumor progression rate was stratified according to the intraoperative CEUS categorization (fig. 5). Although the data regarding the cumulative local tumor progression rate are still preliminary, these results suggest the accuracy of quantitative treatment evaluation using intraoperative CEUS with extracted-overlay fusion imaging.

The primary advantage of extracted-overlay fusion imaging combined with CEUS is that the tumor with a virtual safety margin is visible during and after RFA, and the therapeutic effect can subsequently be evaluated using CEUS immediately after ablation, without the need to perform contrast-enhanced CT. When incomplete ablation is suspected on intraoperative CEUS, additional ablation can be immediately performed. This greatly reduces the patients' 


\section{Liver Cancer}

physical and mental burden, the length of the hospital stay, and the medical expenses associated with RFA treatment (e.g., cost of the RFA electrodes and CT or MR imaging acquisition for treatment evaluation).

The second advantage of extracted-overlay fusion imaging combined with CEUS is that it enables quantitative measurement of the minimal ablative margin. This imaging technique enables the judgment of whether $\mathrm{a} \geq 5$-mm margin can be achieved in addition to the determination of complete ablation of the tumor itself. Thus, the performance of repeat ablation until the tumor and 5-mm virtual margin are completely encompassed by the perfusion defect on CEUS may be an effective strategy to achieve a $\geq 5-\mathrm{mm}$ margin and thereby prevent local tumor progression from the microsatellite lesions around the tumor [26]. In this study, our aim was the complete ablation of the target tumor itself, not the provision of a $\geq 5$-mm margin. Consequently, some tumors showed local tumor progression post-ablation. However, local tumor progression was not observed at all in group III. Therefore, if the therapeutic goal is set as the achievement of a $\geq 5$-mm margin in all cases, the local tumor progression rate will likely be further improved. The third advantage is the noninvasiveness of CEUS. This technique does not cause radiation exposure and can be applied to patients with poor renal function or who are allergic to iodine.

A disadvantage of extracted-overlay fusion imaging is that it is influenced by the tumor location. In the present study, treatment evaluation was impossible using CEUS in 6 HCCs (7.1\%) located far below the body surface because of signal attenuation. Moreover, 5 of 8 HCCs (62.5\%) regarded as incompletely ablated on CT-CT/MR-MR fusion imaging after the first treatment session were located just beneath the diaphragm. Registration of US and CT/ MR reference images might be somewhat inaccurate in this region because of the lack of appropriate landmarks such as branching vessels. Therefore, careful assessment should be performed for HCCs located deep below the body surface or just beneath the diaphragm.

The limitations of this study are its retrospective nature and the inclusion of only a small number of patients. Prospective studies are needed to assess whether this novel evaluation method will eliminate the necessity for contrast-enhanced CT after RFA.

In conclusion, extracted-overlay fusion imaging combined with CEUS is clinically feasible for intraoperative and quantitative evaluation of the effectiveness of RFA treatment and could improve the efficacy of RFA.

\section{Conflict of Interest}

All authors declare that there are no conflicts of interest.

\section{References}

1 Wood BJ, Kruecker J, Abi-Jaoudeh N, Locklin JK, Levy E, Xu S, Solbiati L, Kapoor A, Amalou H, Venkatesan AM: Navigation systems for ablation. J Vasc Interv Radiol 2010;21(Suppl):S257-S263.

2 Krücker J, Xu S, Venkatesan A, Locklin JK, Amalou H, Glossop N, Wood BJ: Clinical utility of real-time fusion guidance for biopsy and ablation. J Vasc Interv Radiol 2011;22:515-524. 
3 Makino Y, Imai Y, Igura T, Ohama H, Kogita S, Sawai Y, Fukuda K, Ohashi H, Murakami T: Usefulness of the multimodality fusion imaging for the diagnosis and treatment of hepatocellular carcinoma. Dig Dis 2012;30:580-587.

4 Kunishi Y, Numata K, Morimoto M, Okada M, Kaneko T, Maeda S, Tanaka K: Efficacy of fusion imaging combining sonography and hepatobiliary phase MRI with Gd-EOB-DTPA to detect small hepatocellular carcinoma. AJR Am J Roentgenol 2012;198:106-114.

5 Makino Y, Imai Y, Ohama H, Igura T, Kogita S, Sawai Y, Fukuda K, Takamura M, Ohashi H, Murakami T: Ultrasonography fusion imaging system increases the chance of radiofrequency ablation for hepatocellular carcinoma with poor conspicuity on conventional ultrasonography. Oncology 2013;84(Suppl 1):44-50.

6 Lee MW, Rhim H, Cha DI, Kim YJ, Choi D, Kim YS, Lim HK: Percutaneous radiofrequency ablation of hepatocellular carcinoma: fusion imaging guidance for management of lesions with poor conspicuity at conventional sonography. AJR Am J Roentgenol 2012;198:1438-1444.

7 Jung EM, Friedrich C, Hoffstetter P, Dendl LM, Klebl F, Agha A, Wiggermann P, Stroszcynski C, Schreyer AG: Volume navigation with contrast enhanced ultrasound and image fusion for percutaneous interventions: first results. PLoS ONE 2012;7:e33956.

8 Makino Y, Imai Y, Igura T, Hori M, Fukuda K, Sawai Y, Kogita S, Ohama H, Matsumoto Y, Nakahara M, Zushi S, Kurokawa M, Isotani K, Takamura M, Fujita N, Murakami T: Utility of computed tomography fusion imaging for the evaluation of the ablative margin of radiofrequency ablation for hepatocellular carcinoma and the correlation to local tumor progression. Hepatol Res 2013;43:950-958.

9 Kim YS, Lee WJ, Rhim H, Lim HK, Choi D, Lee JY: The minimal ablative margin of radiofrequency ablation of hepatocellular carcinoma ( $>2$ and $<5 \mathrm{~cm}$ ) needed to prevent local tumor progression: 3D quantitative assessment using CT image fusion. AJR Am J Roentgenol 2010;195:758-765.

10 Kim KW, Lee JM, Klotz E, Kim SJ, Kim SH, Kim JY, Han JK, Choi BI: Safety margin assessment after radiofrequency ablation of the liver using registration of preprocedure and postprocedure CT images. AJR Am J Roentgenol 2011;196:W565-W572.

11 Tomonari A, Tsuji K, Yamazaki H, Aoki H, Kang JH, Kodama Y, Sakurai Y, Maguchi H: Feasibility of fused imaging for the evaluation of radiofrequency ablative margin for hepatocellular carcinoma. Hepatol Res 2013;43:728-734.

12 Passera K, Selvaggi S, Scaramuzza D, Garbagnati F, Vergnaghi D, Mainardi L: Radiofrequency ablation of liver tumors: quantitative assessment of tumor coverage through CT image processing. BMC Med Imaging 2013;13:3.

13 Makino Y, Imai Y, Igura T, Hori M, Fukuda K, Sawai Y, Kogita S, Fujita N, Takehara T, Murakami T: Comparative evaluation of three-dimensional Gd-EOB-DTPA-enhanced MR fusion imaging with CT fusion imaging in the assessment of treatment effect of radiofrequency ablation of hepatocellular carcinoma. Abdom Imaging 2015;40:102-111.

14 Sakakibara M, Ohkawa K, Katayama K, Imanaka K, Ishihara A, Hasegawa N, Kimura H: Three-dimensional registration of images obtained before and after radiofrequency ablation of hepatocellular carcinoma to assess treatment adequacy. AJR Am J Roentqenol 2014;202:W487-W495. [Medline]

15 Makino Y, Imai Y, Igura T, Kogita S, Sawai Y, Fukuda K, Hori M, Kudo M, Murakami T: Usefulness of the extracted-overlay function in CT/MR-ultrasonography fusion imaging for radiofrequency ablation of hepatocellular carcinoma. Dig Dis 2013;31:485-489.

16 Guibal A, Bertin C, Egels S, Savier E, Grenier PA, Lucidarme 0: Contrast-enhanced ultrasound (CEUS) follow-up after radiofrequency ablation or cryoablation of focal liver lesions: treated-area patterns and their changes over time. Eur Radiol 2013;23:1392-1400.

17 Inoue T, Kudo M, Hatanaka K, Arizumi T, Takita M, Kitai S, Yada N, Hagiwara S, Minami Y, Sakurai T, Ueshima K, Nishida N: Usefulness of contrast-enhanced ultrasonography to evaluate the post-treatment responses of radiofrequency ablation for hepatocellular carcinoma: comparison with dynamic CT. Oncology 2013;84(Suppl 1):51-57.

18 Numata K, Fukuda H, Morimoto M, Kondo M, Nozaki A, Oshima T, Okada M, Takebayashi S, Maeda S, Tanaka K: Use of fusion imaging combining contrast-enhanced ultrasonography with a perflubutane-based contrast agent and contrast-enhanced computed tomography for the evaluation of percutaneous radiofrequency ablation of hy pervascular hepatocellular carcinoma. Eur J Radiol 2012;81:2746-2753.

19 Onishi H, Kim T, Imai Y, Hori M, Nagano H, Nakaya Y, Tsuboyama T, Nakamoto A, Tatsumi M, Kumano S, Okada M, Takamura M, Wakasa K, Tomiyama N, Murakami T: Hypervascular hepatocellular carcinomas: detection with gadoxetate disodium-enhanced MR imaging and multiphasic multidetector CT. Eur Radiol 2012;22:845-854.

20 Nakazawa T, Kokubu S, Shibuya A, Ono K, Watanabe M, Hidaka H, Tsuchihashi T, Saigenji K: Radiofrequency ablation of hepatocellular carcinoma: correlation between local tumor progression after ablation and ablative margin. AJR Am J Roentgenol 2007;188:480-488.

21 Koda M, Tokunaga S, Miyoshi K, Kishina M, Fujise Y, Kato J, Matono T, Okamoto K, Murawaki Y, Kakite S: Assessment of ablative margin by unenhanced magnetic resonance imaging after radiofrequency ablation for hepatocellular carcinoma. Eur J Radiol 2012;81:2730-2736.

22 Mori K, Fukuda K, Asaoka H, Ueda T, Kunimatsu A, Okamoto Y, Nasu K, Fukunaga K, Morishita Y, Minami M: Radiofrequency ablation of the liver: determination of ablative margin at MR imaging with impaired clearance of ferucarbotran-feasibility study. Radiology 2009;251:557-565.

23 Koda M, Tokunaga S, Miyoshi K, Kishina M, Fujise Y, Kato J, Matono T, Murawaki Y, Kakite S, Yamashita E: Ablative margin states by magnetic resonance imaging with ferucarbotran in radiofrequency ablation for hepatocellular carcinoma can predict local tumor progression. J Gastroenterol 2013;48:1283-1292. 
24 Okubo H, Kokubu S, Komiyama M, Yamagata H, Uchiyama A, Miyazaki A, Watanabe S: Radiofrequency ablation of hepatocellular carcinoma: the feasibility of magnetic resonance imaging with gadolinium ethoxybenzyl diethylene triamine pentaacetic acid for evaluating the ablative margin. Hepatol Res 2010;40:10341041.

25 Nishikawa H, Inuzuka T, Takeda H, Nakajima J, Sakamoto A, Henmi S, Matsuda F, Eso Y, Ishikawa T, Saito S, Kita R, Kimura T, Osaki Y: Percutaneous radiofrequency ablation therapy for hepatocellular carcinoma: a proposed new grading system for the ablative margin and prediction of local tumor progression and its validation. J Gastroenterol 2011;46:1418-1426.

26 Okusaka T, Okada S, Ueno H, Ikeda M, Shimada K, Yamamoto J, Kosuge T, Yamasaki S, Fukushima N, Sakamoto M: Satellite lesions in patients with small hepatocellular carcinoma with reference to clinicopathologic features. Cancer 2002;95:1931-1937. 